\title{
Premixed Turbulent Flames
}

\author{
Andrei N. Lipatnikov, ${ }^{1}$ Alexey A. Burluka, ${ }^{2}$ Tatsuya Hasegawa, ${ }^{3}$ and Vladimir A. Sabel'nikov ${ }^{4}$ \\ ${ }^{1}$ Department of Applied Mechanics, Chalmers University of Technology, 41296 Gothenburg, Sweden \\ ${ }^{2}$ School of Mechanical Engineering, The University of Leeds, Woodhouse Lane, Leeds LS2 9JT, UK \\ ${ }^{3}$ Center for Interdisciplinary Studies on Resource Recovery and Refinery in Asia (CISRA), EcoTopia Science Institute, \\ F3-4 (670), Nagoya University, Nagoya 464-8603, Japan \\ ${ }^{4}$ ONERA/DEFA, Centre de Palaiseau, Chemin de la Hunière, 91761 Palaiseau, France
}

Correspondence should be addressed to Andrei N. Lipatnikov, lipatn@chalmers.se

Received 23 November 2011; Accepted 23 November 2011

Copyright (c) 2011 Andrei N. Lipatnikov et al. This is an open access article distributed under the Creative Commons Attribution License, which permits unrestricted use, distribution, and reproduction in any medium, provided the original work is properly cited.

Turbulent combustion of premixed gases is widely used for energy conversion in transportation, for example, piston engines in cars and light aircrafts, aero-engine afterburners, and stationary power generation, for example, gas turbines. Current environmental and efficiency challenges call urgently for new solutions that satisfy stringent requirements for ultra low emissions as well as highly efficient combustion technology. To make any significant progress in this area, both fundamental and applied studies of numerous unresolved issues relevant to premixed turbulent flames are strongly necessary. This special issue contains seven papers aimed at contributing to fundamental understanding of premixed turbulent combustion as well as to more efficient use of this process in future engines.

Since the early days of research into premixed turbulent flames, turbulent burning velocity or flame speed was in the focus of numerous experimental, theoretical, and numerical studies. Despite long-term extensive research, certain basic issues have not yet been resolved and some of them are addressed in the first three contributions. In the paper entitled "Determination of the burning velocity domain of a statistically stationary turbulent premixed flame in presence of counter-gradient transport," the classical academic problem of the burning velocity in a fully developed statistically stationary, planar, and one-dimensional turbulent flame is theoretically analyzed in order to investigate the influence of countergradient scalar transport (i.e., transport of combustion products towards the burned side of the flame, caused by pressure gradient induced by the heat release) on the burning velocity. In particular, it is demonstrated that, even if turbulent scalar transport shows the countergradient behavior at the cold boundary, "there still exists a possibility of observing a steady regime of propagation." The paper entitled "Burning rate in impinging jet flames" deals also with the problem of countergradient turbulent scalar transport by invoking a recently developed simple model of this phenomenon in order to validate a method for evaluating turbulent burning velocity proposed earlier based on a theoretical consideration. The influence of hydrodynamic effects caused by heat release on turbulent burning velocity is also discussed in the paper entitled "Influence of turbulent scalar mixing physics on premixed flame propagation," which highlights another effect of that kind, that is, dilatation, rather than countergradient transport.

Direct numerical simulation (DNS) is a rapidly developing powerful numerical tool for fundamental research into turbulent flows and turbulent flames, in particular. The present special issue contains three contributions relevant to DNS of premixed turbulent combustion. In the paper entitled "Effects of turbulent Reynolds number on the displacement speed statistics in the thin reaction zones regime of turbulent premixed combustion," a DNS database obtained from premixed turbulent flames characterized by different Reynolds numbers is analyzed in order to investigate statistical characteristics of the instantaneous flame front (its local displacement speed, surface density, curvature, and strain rate) as well as relations between these statistical characteristics and the influence of the Reynolds number on them. In the paper entitled "Statistics of conditional fluid velocity in the corrugated flamelets regime of turbulent premixed combustion: 
a direct numerical simulation study," a single DNS database is considered in order to study the behavior of the first and second moments of turbulent velocity field, conditioned either to unburned mixture, or to burned mixture, or to the instantaneous flame front. In particular, it is shown that flow velocities conditioned within unburned or unburned side of the front are approximately equal in the largest part of turbulent flame brush with the exception of its leading edge. In the paper entitled "Direct numerical simulations of the impact of high turbulence intensities and volume viscosity on premixed methane flames," DNS data obtained from highintensity turbulent premixed flames are reported, and it is argued that, contrary to hydrogen-air flames, volume viscosity weakly affects methane-air flames and may be ignored in DNS research into turbulent combustion of hydrocarbon-air mixtures.

While the above six papers address basic issues of premixed turbulent combustion either theoretically or numerically, the paper entitled "Effects of turbulence on the combustion properties of partially premixed flames of canola methyl ester and diesel blends" is an example of an applied experimental study aimed at developing clean combustion technology by utilizing an alternative biofuel canola methyl ester (CME). The paper reports measured data on the influence of turbulence on emissions from partially premixed flames of blends of CME and a Diesel fuel.

\author{
Andrei N. Lipatnikov \\ Alexey A. Burluka \\ Tatsuya Hasegawa \\ Vladimir A. Sabel'nikov
}



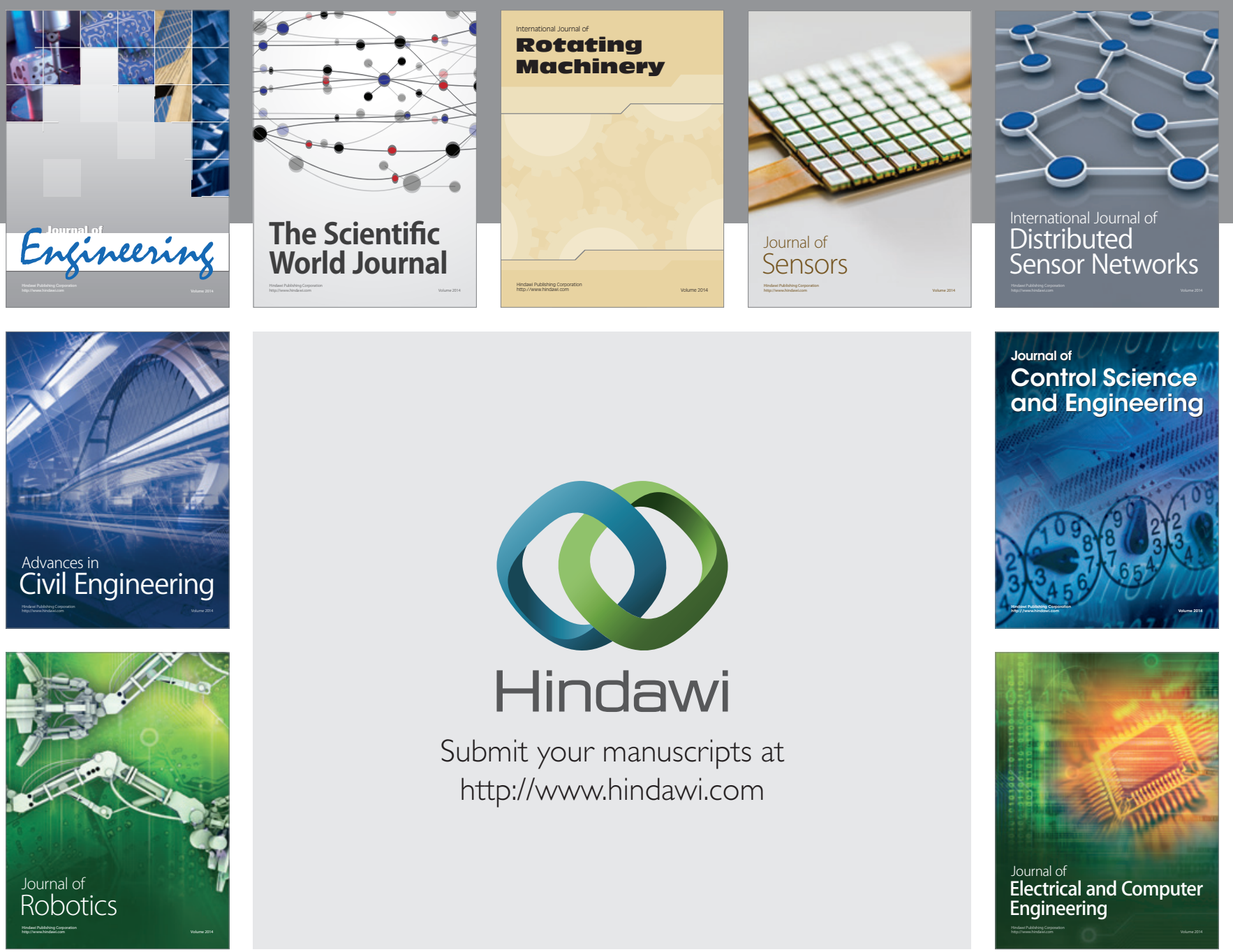

Submit your manuscripts at

http://www.hindawi.com
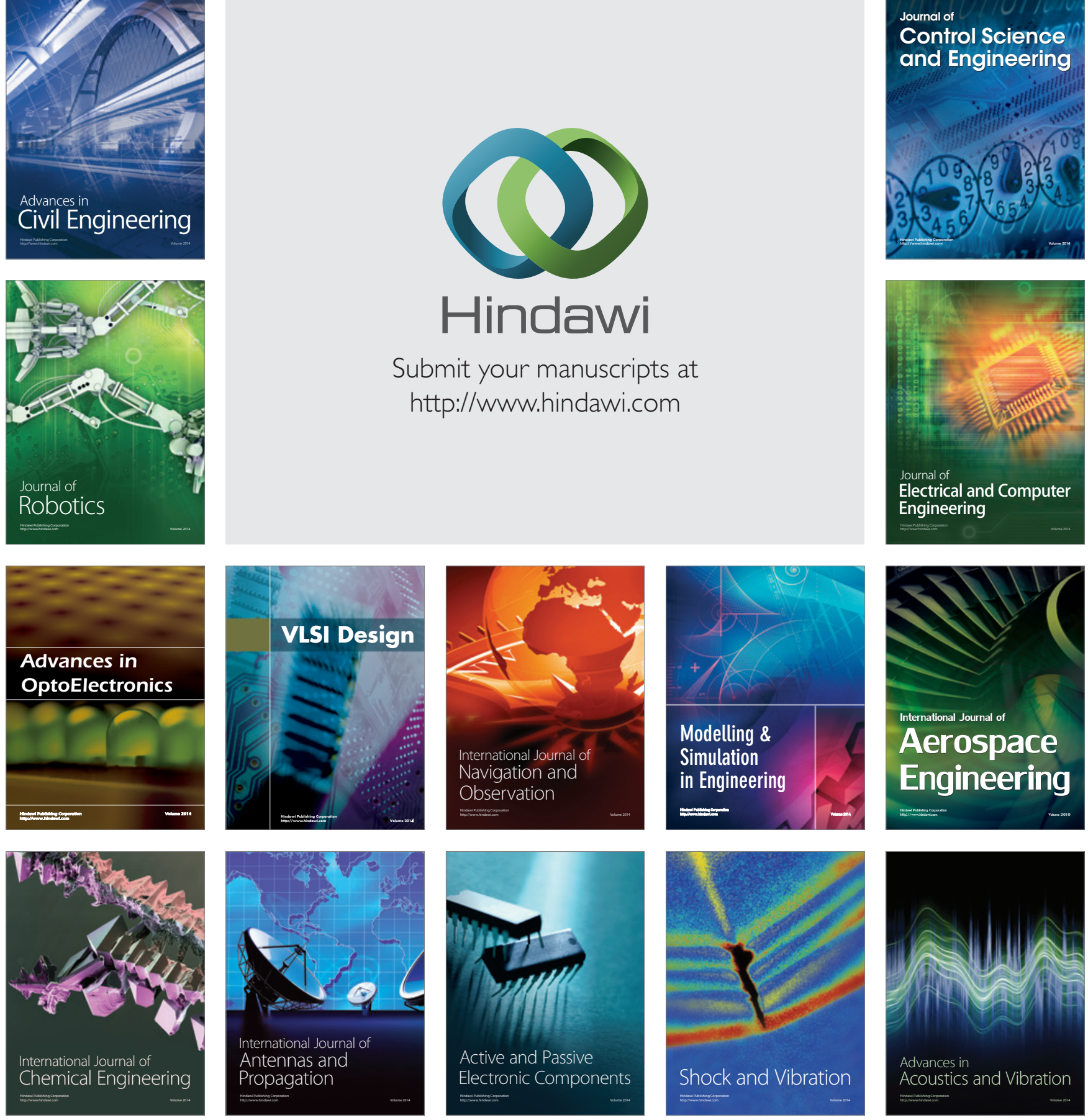